\title{
'Ozone Nano Bubble Water: A Magic Wand for the Treatment of Periodontal Disease'
}

\author{
Dr. Manoj Kumar
}

\begin{abstract}
Chronic periodontitis is a highly prevalent disease affecting mainly the middle aged population. Traditionally a lot of materials have been used as an adjunct to scaling and root planing for the treatment of periodontal disease with variable success. The present manuscript highlights the efficacy of a novel product, ozone nano bubble water (NBW3) in the treatment of periodontal disease, which could be a promising antimicrobial agent and may revolutionize the field of periodontology as well of pharmacy in the management of periodontitis.
\end{abstract}

Keywords: Ozone nano bubble water, periodontitis, $P$ gingivalis, A actinomycetemcomitans

\section{INTRODUCTION}

Chronic periodontitis is an inflammatory disease of the periodontium caused by microorganisms present in the dental plaque resulting in progressive bone and attachment loss of the tooth. ${ }^{1}$ Loss of connective tissue attachment to the teeth is the most important clinical feature which differentiates periodontitis from gingivitis. ${ }^{2}$ The periodontopathic bacteria are mainly anaerobic and Porphyromonas gingivalis and Aggregatibacter actinomycetemcomitans have been identified as the key perpetuators of periodontal disease and the primary goal of periodontal treatment is to eliminate them.

Mechanical debridement by scaling and root planing (SRP) is still considered the gold standard and the mainstay of all periodontal treatment procedures. However, complete mechanical debridement of the deep periodontal pockets is difficult to accomplish. ${ }^{3}$ This may fail to completely eliminate the pathogenic bacteria situated within the gingival tissues or in areas where instrumentation is difficult. Hence, lot of chemotherapeutic agents namely, systemic antibiotics, antiseptic mouthwashes, local drug delivery agents have been used as an adjunct to mechanical debridement with varying degrees of success. These agents have their share of disadvantages like drug resistance, toxicity and hypersensitivity. Therefore, the search for newer and safer agents is still ongoing. Of late, ozone has garnered considerable interest in dentistry.

\section{Ozone Nano Bubble Water}

Ozone was discovered by Schonbein in 1840 and has been used widely in industry. ${ }^{4}$ Ozone is a triatomic molecule and exists as a colorless gas with a pungent odour at room temperature, detectable at concentrations as low as 0.02 to $0.05 \mathrm{ppm}$ (by volume). ${ }^{5}$ Ozone is being used in dentistry owing to its antimicrobial and disinfectant properties.It has strong antimicrobial action against bacteria, fungi, protozoa and viruses without inducing microbial resistance. It is highly biocompatible with fibroblasts, cementoblasts as well as epithelial cells.

Periodontal application of ozone is usually done in gaseous, aqueous and oil forms. Ozone has been claimed to inhibit the growth of Porphyromonas gingivalis, Aggregatibacter actinomycetemcomitans and Tanerella forsythia, the main etiological agents for periodontal disease. ${ }^{6,8}$ Ozonized water has been successfully used as a subgingival irrigant in the treatment of chronic and aggressive periodontitis as well as to reduce inflammation in patients undergoing orthodontic treatment.

However, ozonated water has a half-life of only 20 minutes and has to be used within 5-10 minutes of its production. To overcome this, a patented product, ozone nano bubble water(NBW3) was developed by CHIBA and TAKAHASHI. ${ }^{9} \mathrm{~A}$ nano-bubble is less than $100 \mathrm{~nm}$ in diameter, and is produced by the collapse of a micro-bubble ( $\leqslant 50 \mu \mathrm{m}$ in diameter) in an electrolyte solution by means of a physical stimulus (such as a shock wave caused by electrical discharge in the solution). The physical properties of nano- bubble are inferred from those of micro-bubble. ${ }^{10}$ The positive ions in electrolyte solution become concentrated around the gas nucleus due to its negatively charged surface 
and act as shells that prevent the gas from dispersing (the salting out phenomenon). Due to this behavior, nano-bubbles remain stable for more than 6 months in electrolyte solution if it is protected against exposure to ultraviolet rays. The high stability of NBW3 allows for bottling and use as a disinfectant solution.

In a pioneer study performed by $\mathrm{S}$ Hayakumo et $\mathrm{al}^{11}$,they used nano bubble water to examine its bactericidal effects on $\mathrm{P}$ gingivalis and $\mathrm{A}$ actinomycetemcomitans using an in vitro time kill study. They found NBW3 to be more effective than chlorhexidine as an adjunct for periodontal treatment and within 0.5 minutes of exposure, $\mathrm{P}$ gingivalis and $\mathrm{A}$ actinomycetemcomitans levels dropped to less than lower limits of detection $(<10$ CFUs $\mathrm{mL}-1)$. Furthermore, ozone nano bubble water was biocompatible and did not induce cytotoxic effects on the oral tissues using $3 \mathrm{D}$ buccal and gingival tissue models.

Although the results obtained in the above mentioned study are remarkable, it cannot be directly implied in clinical practice, as the study was an in vitro study. Secondly, treatment of periodontal disease involves dealing with plaque as a biofilm, which was not done in the above mentioned study. Thirdly, findings of in vitro studies cannot be implied in the oral cavity as antiseptics are diluted because of the presence of saliva and their efficacy in the subgingival area decreases because of constant outflow of gingival crevicular fluid and binding of antiseptic to serum proteins.

\section{Conclusion}

Hence, more studies using this novel product are warranted wherein it is used as an adjunct with scaling and root planing to explore its possible role and efficacy in the treatment of periodontal diseases.

\section{REFERENCES}

[1] Kornman KS. Mapping the pathogenesis of periodontitis: A New Look. J Periodontol 2008; 79:1560- 8 .

[2] Informational Paper: The pathogenesis of periodontal diseases. J Periodontol 1999; 70:450- 70.

[3] Cobb CM. Non surgical pocket therapy: Mechanical. Ann Periodontol 1996; 1:443-90.

[4] Lynch, E. (2004) Ozone: The revolution in dentistry. Quintessence Publishing Co Ltd., Surrey.

[5] United States Environmental Protection Agency (1999) Alternative disinfectants and oxidants. EPA, 815-R-99- 014.

[6] Azarpazhooh A, Limeback H.The application of ozone in dentistry: a systematic review of literature.J Dent. 2008; 36(2):104-16.

[7] Schlagenhauf U, Horlacher V, Netuschil L, Brecx M. The effect of repeated subgingival oxygen irrigations in previously untreated deep periodontal pockets. J ozone versus conventional irrigation. Clin Periodontol. 1994; 21:48-50.

[8] Sae Hayakumo, Shinichi Arakawa, Masayoshi Takahashi, Keiko Kondo, Yoshihiro Manoand Yuichi Izumi. Effects of ozone nano-bubble water on periodontopathic bacteria and oral cells - in vitro studies.Sci. Technol. Adv. Mater. 2014; 15: 055003.

[9] Chiba K and Takahashi M 2008 Nano-bubble production method JAPAN PATENT P4144669. 2008-09-03.

[10] Hirose Y1, Yasui T, Taguchi K, Fujii Y et al. Oxygen nano-bubble water reduces calcium oxalate deposits and tubular cell injury in ethylene glycol-treated rat kidney. Urolithiasis 2013 Aug; 41(4):279-94.

[11] Sae Hayakumo, Shinichi Arakawa, Masayoshi Takahashi, Keiko Kondo et al. Effects of ozone nano-bubble water on periodontopathic bacteria and oral cells - in vitro studies. Sci. Technol. Adv. Mater. 15 (2014) 055003. 